\title{
Actin Capping Protein Is Required for Dendritic Spine Development and Synapse Formation
}

\author{
Yanjie Fan ${ }^{1}$, Xin Tang ${ }^{2}$, Eric Vitriol ${ }^{1}$, Gong Chen ${ }^{2}$, and James Q. Zheng ${ }^{1}$ \\ ${ }^{1}$ Departments of Cell Biology and Neurology, Center for Neurodegenerative Diseases, Emory University School of Medicine, Atlanta, Georgia 30322, and \\ ${ }^{2}$ Department of Biology, Huck Institutes of Life Sciences, Pennsylvania State University, University Park, Pennsylvania 16802
}

Dendritic spines serve as the postsynaptic platform for most excitatory synapses in the mammalian brain, and their shape and size are tightly correlated with synaptic strength. The actin cytoskeleton plays a crucial role in the spine structure and its modifications during synapse development and plasticity, but the underlying regulatory mechanisms remain to be elucidated. Here, we report that actin capping protein (CP), a regulator of actin filament growth, plays an essential role for spine development and synapse formation. We found that $\mathrm{CP}$ expression in rat hippocampus is elevated at and after the stage of substantial synapse formation. CP knockdown in hippocampal cultures resulted in a marked decline in spine density accompanied by increased filopodia-like protrusions. Moreover, the spines of CP knockdown neurons exhibited an altered morphology, highlighted by multiple thin filopodia-like protrusions emerging from the spine head. Finally, the number of functional synapses was reduced by CP knockdown as evidenced by a reduction in the density of paired presynaptic and postsynaptic markers and in the frequency of miniature EPSCs. These findings indicate that capping of actin filaments by $\mathrm{CP}$ represents an essential step for the remodeling of the actin architecture underlying spine morphogenesis and synaptic formation during development.

\section{Introduction}

Most of the excitatory synapses in the vertebrate brain reside on dendritic spines that provide the platform for postsynaptic specializations, including clustered synaptic receptors. During development, dendritic spines are formed upon axonal contact (Yoshihara et al., 2009), and their shape and size are tightly correlated with synaptic strength (Yuste and Bonhoeffer, 2001; Kasai et al., 2003). Abnormality in spine number and shape has been observed in a number of neurological disorders and contributes to brain dysfunction (van Spronsen and Hoogenraad, 2010). Actin is the major cytoskeletal component in dendritic spines and provides the structural basis for spine formation, maturation, modification, and elimination during synaptic development, plasticity, and dysfunction (Tada and Sheng, 2006; Cingolani and Goda, 2008; Hotulainen and Hoogenraad, 2010). Importantly, the actin cytoskeleton, in conjunction with other scaffolding molecules, establishes the cytoarchitecture for subcellular organization of postsynaptic components that ensures effective postsynaptic signaling (Carlisle and Kennedy, 2005).

Received Jan. 7, 2011; revised March 28, 2011; accepted April 24, 2011.

Author contributions: J.Q.Z. designed research; Y.F. and X.T. performed research; E.V. contributed unpublished reagents/analytic tools; Y.F., X.T., G.C., and J.Q.Z. analyzed data; Y.F. and J.Q.Z. wrote the paper.

This research was supported by National Institutes of Health Grants GM083889, GM084363, and HD023315 (to J.Q.Z.) and MH083911 (to G.C.), as well as in part by P30NS055077 to the Neuronal Imaging Core of Emory Neuroscience. We thank Dr. John Hammer and Kirsten Remmert (National Heart Lung and Blood Institute, National Institutes of Health, Bethesda, MD) for providing the antibody against the $\beta$ subunit of capping protein and sharing the staining protocol. We also thank Dr. James Bear (University of North Carolina, Chapel Hill, NC) for sharing the constructs for capping protein manipulation.

Correspondence should be addressed to James Q. Zheng, Department of Cell Biology, Emory University School of Medicine, 615 Michael Street, Atlanta, GA 30322. E-mail: james.zheng@emory.edu.

DOI:10.1523/JNEUROSCI.0115-11.2011

Copyright $\odot 2011$ the authors $\quad 0270-6474 / 11 / 3110228-06 \$ 15.00 / 0$
The actin cytoskeleton and its dynamics are regulated by a broad array of binding proteins, but whether and how they function together in dendritic spines remain to be elucidated (Hotulainen and Hoogenraad, 2010). Capping protein (CP) binds the barbed ends of actin filaments to prevent their elongation and facilitate Arp2/3-mediated nucleation (Akin and Mullins, 2008). $\mathrm{CP}$ exists and functions as an $\alpha / \beta$ heterodimer, and a single subunit is unstable (Cooper and Sept, 2008). Although both $\alpha 1$ and $\alpha 2$ subunits are abundant in most tissues (Hart et al., 1997), $\beta 2$ is the predominant $\beta$ subunit in the brain (Schafer et al., 1994). A recent study showed that $\mathrm{CP}$ is present in dendritic spines of cultured hippocampal neurons and the branched actin filament network containing CP appears to be a prominent feature of the spine head (Korobova and Svitkina, 2010). However, a role for $\mathrm{CP}$ in dendritic spines and synaptic functions has not been established. In this study, we examined the role of CP in spine development and synapse formation. Our data show that CP is essential for spine development and maturation leading to functional synapses.

\section{Materials and Methods}

Constructs and reagents. DNA constructs of pmOrange, pEGFP-N1, and pEGFP-tubulin were from Clontech, and the mOrange sequence from pmOrange was subcloned into pEGFP-N1 (Gu et al., 2008). EGFPCP $\beta 2$, CPshRNA-mRFP, and CPshRNA-GFP-CP [knockdown rescue $(\mathrm{KDR})]$ have been described previously (Mejillano et al., 2004; Vitriol et al., 2007), and CPshRNA targets both mouse and rat CP $\beta 2$. Anti-CP $\beta 2$ ( $\mathrm{mAb} 3 \mathrm{~F} 2.3)$, anti- $\mathrm{CP} \alpha 1$, anti- $\mathrm{CP} \alpha 2$ (mAb 5B12.3), and anti-SV2 were from the Developmental Studies Hybridoma Bank at the University of Iowa. Other antibodies used were antitubulin antibody (PRB-435P; Covance) and anti-PSD-95 (MA1-045; Thermo Scientific). A custommade antibody against the $\mathrm{C}$ terminus of the $\mathrm{CP} \beta$ subunit (Schafer et 
al., 1994) was used for immunostaining (generously provided by Dr. John Hammer of the National Heart Lung and Blood Institute, National Institutes of Health, Bethesda, MD).

Neuronal culture, transfection, and imaging. Rat hippocampal cultures and live imaging were performed as described previously ( $\mathrm{Gu}$ et al., 2008). Cells were transfected using the calcium phosphate transfection kit (Clontech) at DIV13 and imaged between DIV21 and DIV23. Live imaging was performed using a Nikon C1 confocal system. Typically, a $3 \mathrm{D}$ stack of images of a dendritic region was acquired and then projected into a $2 \mathrm{D}$ image (maximal intensity) for visualization and analysis. The 3D images of spines were reconstructed using the "Surpass" function of Imaris 7.2 (Andor Technology). Filopodia were defined as thin protrusions without a distinguishable head, and spines were defined as protrusions with a length $<4 \mu \mathrm{m}$ and an expanded distinguishable head (head/ neck ratio $>1.2$ ) (Hotulainen et al., 2009). Spine and filopodia numbers were counted manually to calculate the density (number per unit length of the parent dendrite). Spine head width was measured as spine diameter (the longest possible axis), and neck length was from proximal edge of the spine head to the edge of the dendrite. For spines with no discernible necks, a minimum value of $0.2 \mu \mathrm{m}$ was used. To quantify the synaptic density, the cluster number and area of SV2 and PSD-95 per unit neurite length were counted and measured using ImageJ (National Institutes of Health). Fluorescence recovery after photobleaching (FRAP) was done using a Nikon A1R confocal system. Photobleaching was achieved by scanning only the spine of interest for $500 \mathrm{~ms}$ with $100 \%$ power of 488 $\mathrm{nm}$ laser ( $\sim 2 \mathrm{~mW}$ at the $60 \times / 1.49$ objective), and subsequent imaging of fluorescence recovery was done by scanning region of interest with $2 \%$ laser power at a $3 \mathrm{~s}$ interval for $\sim 3 \mathrm{~min}$.

Western blot. Hippocampi from E18, P9, and P18 rats of both sexes were snap-frozen in liquid nitrogen and then homogenized and extracted with RIPA lysis buffer [50 mm Tris- $\mathrm{HCl}, 150 \mathrm{~mm} \mathrm{NaCl}, 1 \%$ (v/v) Triton $\mathrm{X}-100,0.25 \%(\mathrm{w} / \mathrm{v})$ Na-deoxycholate, $1 \mathrm{~mm}$ EDTA, pH 7.4] supplemented with protease inhibitor mixture (Sigma P2850). Extracts were dissolved in NuPage sample buffer (Invitrogen) with $50 \mathrm{~mm}$ DTT and heated at $85^{\circ} \mathrm{C}$ for $5 \mathrm{~min}$. Equal amounts of protein as determined by BCA measurement were loaded and fractioned by SDS-PAGE in a $10 \%$ acrylamide gel and subsequently transferred to nitrocellulose membrane. Membranes were treated with 5\% milk in PBS buffer with $0.05 \%$ Tween 20 and then blotted with primary antibody. Bound antibodies were detected by HRP-conjugated secondary antibody (Jackson ImmunoResearch), visualized by chemiluminescence using ECL (Pierce), and quantified using the gel analysis routine of ImageJ software.

Immunostaining. Neurons were fixed for $10 \mathrm{~min}$ with $4 \%(\mathrm{w} / \mathrm{v})$ paraformaldehyde and permeabilized with $0.1 \%(\mathrm{w} / \mathrm{v})$ Triton X-100. After blocking with 5\% (w/v) BSA in PBS for $1 \mathrm{~h}$, cells were incubated with primary antibodies overnight at $4^{\circ} \mathrm{C}$, followed by $1 \mathrm{~h}$ labeling with fluorescent secondary antibody or fluorescent phalloidin.

Electrophysiology. Whole-cell patch-clamp recordings were performed in mouse cortical cultures using a Multiclamp 700A amplifier (Molecular Devices) as described previously (Deng et al., 2007; Gu et al., 2010). Similarly, cells were transfected using the calcium phosphate method at DIV13-14, and fluorescent cells were identified for patch-clamp recording at DIV21. The membrane potential was held at $-70 \mathrm{mV}$. Data were acquired using pClamp9 software, sampled at $5 \mathrm{kHz}$, and filtered at 1 $\mathrm{kHz}$. The miniature EPSCs (mEPSCs) were recorded with the addition of $0.5 \mu \mathrm{M}$ TTX and $100 \mu \mathrm{M}$ picrotoxin to block action potentials and $\mathrm{GABA}_{\mathrm{A}}$ receptors.

\section{Results}

\section{$\mathrm{CP}$ expression increases in postnatal hippocampi and accumulates in dendritic spines}

To gain insights into CP functions in brain development, we performed Western blot analysis to examine CP expression in rat hippocampus at E18, P9, and P18, which correspond approximately to stages before, during, and after extensive synapse formation (Fiala et al., 1998). Because the CP $\beta 1$ subunit was not detected in brain (Schafer et al., 1994), we examined only the $\beta 2$ subunit and both $\alpha$ subunits. We found that $\alpha$ and $\beta 2$ subunits exhibited an increasing trend of expression over the course of hippocampal development (Fig. 1A): the CP level increased slightly at P9, but significantly at P18 over E18 $(\mathrm{CP} \alpha 1$ and $\mathrm{CP} \alpha 2: p<0.001$ for P18 vs E18 and P18 vs P9; CP $\beta 2: p<0.001$ for all the pairwise comparisons; ANOVA Turkey test). These data suggest that CP functions during synapse development and refinement.

We next examined the subcellular distribution of CP in primary cultured hippocampal neurons. Consistent with the previous study (Korobova and Svitkina, 2010), we found that CP is highly present in dendritic spines as evidenced by immunostaining using a specific antibody against $\mathrm{CP} \beta 2$ (Fig. $1 B$ ) (Schafer et al., 1994). Furthermore, our live cell imaging of EGFP-tagged $\beta 2$ (hereafter referred to as GFP-CP) showed that CP is highly enriched in the dendritic spines of DIV21 hippocampal neurons compared with the volume marker mOrange (Fig. 1C). The spine localization of $\mathrm{CP}$ is better depicted by ratiometric normalization of GFP-CP to mOrange, because the GFP-CP/mOrange ratio in spines is $2.4 \pm 0.6$ (mean $\pm 95 \%$ confidence interval, from five neurons over 100 spines) when normalized against that of the adjacent dendritic shaft. For neurons expressing GFP and mOrange, the ratio of $\mathrm{GFP} / \mathrm{mOrange}$ in spines is approximately the same as that in dendritic shaft $(1.0 \pm 0.1$, from four neurons over 100 spines). The localization of GFP-CP in spines is further supported by FRAP. Compared with GFP, GFP-CP exhibited a much slower rate of recovery (halftime of $\sim 20 \mathrm{~s}$ for GFP-CP vs $\sim 2 \mathrm{~s}$ for GFP) with $\sim 12 \%$ GFP-CP fluorescence not recovered at the end of recording (Fig. 1D). These data suggest that $\mathrm{CP}$ is preferentially localized to spines, potentially through interactions with other spine components.

\section{CP knockdown impairs spine formation and morphogenesis}

To investigate the function of CP in spine development, we expressed an shRNA that specifically targets CP $\beta 2$ of both mouse and rat (Mejillano et al., 2004). This shRNA construct also encodes mRFP for identification of neurons expressing shRNA. We first verified the effectiveness of CP knockdown by this shRNA in cultured mouse CAD neuroblastoma cells (Qi et al., 1997). Consistently, the endogenous $\mathrm{CP} \beta 2$ level was dramatically reduced $3 \mathrm{~d}$ after shRNA transfection as evidenced by Western blotting (Fig. $2 A$ ). Effective knockdown of endogenous $\mathrm{CP} \beta 2$ in cultured hippocampal neurons was verified by immunostaining (Fig. $2 A$ ). The average intensity of the $\mathrm{CP} \beta 2$ immunofluorescence in shRNA-expressing neurons (identified by the presence of mRFP fluorescence) was $53 \pm 4 \%$ of that of nontransfected neurons in the same dish ( shRNA neurons, 15; nontransfected cells, $>120$ from three different batches of experiments). These results thus confirmed the effectiveness of the CPshRNA for CP $\beta 2$ knockdown in hippocampal neurons.

To examine the effects of CP $\beta 2$ knockdown on spine development, we transfected cultured hippocampal neurons at DIV13 and performed confocal imaging on spines at DIV21. In control neurons, a large portion of dendritic spines has developed into the mushroom shape with a distinct spine head and neck (Fig. $2 B$, arrows) and some have acquired stubby (Fig. $2 B$, arrowheads) as well as thin spine morphologies (Fig. $2 B$, double arrows). In neurons expressing CPshNRA, however, a large percentage of dendritic protrusions exhibited a thin filopodia-like morphology (Fig. $2 B$, red arrows), some of which were branched (Fig. $2 B$, red arrowheads). Most notably, some spine-like protrusions that did form displayed an aberrant morphology. Unlike the bulbous spine heads observed in control neurons, these spine heads appeared to be larger and irregularly shaped and, most intriguingly, 

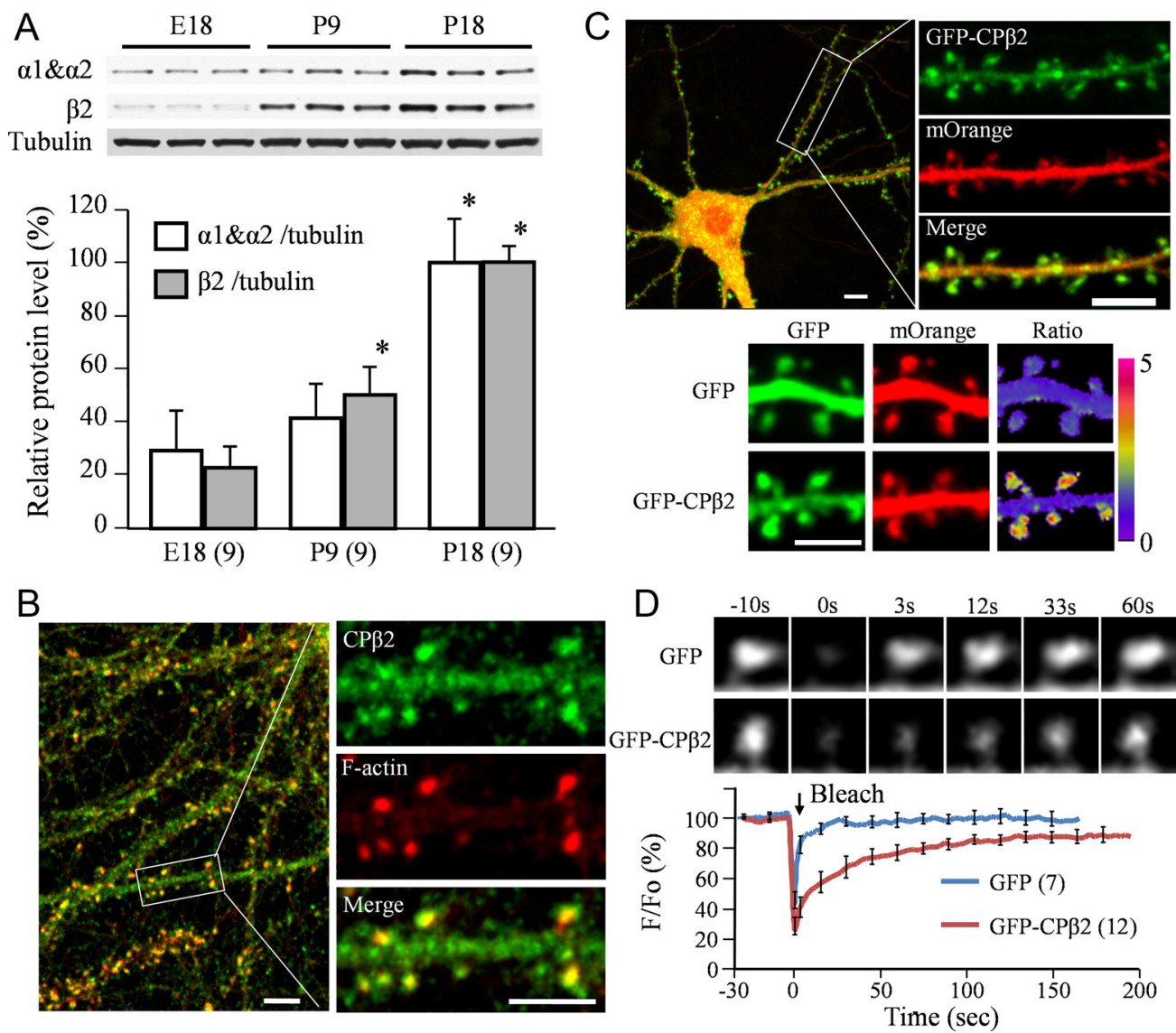

Figure 1. Expression profile and cellular distribution of CP. A, Western blots of $\mathrm{CP} \alpha$ and $\mathrm{CP} \beta$ subunits in E18, $\mathrm{P9}$, and P18 rat hippocampi. Quantification is shown in the bar graph. $\boldsymbol{B}$, Immunostaining of endogenous $C P \beta 2$ together with phalloidin labeling of dendritic spines. C, Subcellular distribution of GFP-CP $\beta 2$ expressed in hippocampal neurons with m0range as the volume marker (top). The GFP-CP or GFP signals were normalized against the m0range signals to generate ratiometric images in pseudocolors (bottom). D, FRAP of GFP and GFP-CP in dendritic spines. Two sample time-lapse sequences are shown to demonstrate the recovery of fluorescence in spine. The curves depict the average intensity at different time points normalized to the first frame. Error bars represent the $95 \%$ confidence interval. Scale bars, $5 \mu \mathrm{m}$. Numbers in parentheses indicate the number of animals $(\boldsymbol{A})$ or cells $(\boldsymbol{D})$ for each group.

had thin filopodia protruding out (Fig. $2 \mathrm{~B}$, asterisks). The abnormality in spine shape and size can be better appreciated by $3 \mathrm{D}$ reconstruction of dendritic protrusions (Fig. 2C). The CPshRNA effects on spines appear to be a direct result of CP $\beta 2$ knockdown because KDR experiments using a construct encoding both CPshRNA and a shRNA-refractory CP mutant (Vitriol et al., 2007) produced dendritic spines similar to that of the control cells (Fig. $2 B$ ). Furthermore, expression of GFP-CP did not seem to perturb the spine morphology. When quantified, the number of total protrusions stemming from the dendritic shaft showed no difference among different groups (Fig. 2D). However, the numbers of spine-like protrusions in $\mathrm{CP} \beta 2$ knockdown cells were significantly reduced (Fig. $2 D ; p<0.05$ compared with the control; ANOVA Dunnett's method), whereas the number of filopodia was much higher than that of control cells (Fig. 2D). Further analysis of single spine size showed that the spine head width and neck length in the CPshRNA group were larger than that of the control (Fig. $2 E ; p<0.01$ and $<0.05$, respectively; rank sum test). These results indicate that $\mathrm{CP}$ is required for the formation and morphogenesis of dendritic spines.

Similar to control cells, dendritic protrusions in CP knockdown cells are actin-based as evidenced by their concentration of F-actin (Fig. 3A). In particular, the filopodia-like protrusions from the spine head are highlighted by F-actin (see magnified panels in Fig. 3A). We found that most of the dendritic protrusions in CP knockdown cells did not contain microtubules except a small fraction of spines (Fig. $3 A$ ), similar to that reported previously (Gu et al., 2008; Hu et al., 2008). To further understand the effects of CP knockdown on spine formation and morphogenesis, we performed confocal imaging to examine the spine development at different days after the transfection of CPshRNA constructs. We found that filopodia were predominant in DIV14 cells with few spine-like protrusions, but a marked number of spines started to emerge from DIV17 to DIV21 (Fig. 3B). In CPshRNA-expressing cells, the thin filopodia-like protrusions were obvious by DIV17 and became predominant by DIV21, together with the large protrusions with thin filopodia-like protrusions on the spine head. We quantified the number of dendritic protrusions (Fig. 3B) and found that control cells underwent an increase in spine formation accompanied by a decrease in the number of filopodia from DIV14 to DIV21 (Fig. 3B). However, in $\mathrm{CP} \beta 2$ knockdown cells, the number of thin filopodia-like protrusions increased over the same period of time, whereas the number of spine-like structures remained unchanged. Together, these data suggest that $\mathrm{CP}$ is involved in spine formation and likely plays a role in spine head expansion.

\section{CP knockdown affects presynaptic and postsynaptic} specialization and synaptic transmission

To examine the formation of functional synapses, we first performed immunostaining to identify presynaptic and postsynaptic specializations using antibodies against SV2 (presynaptic marker) 

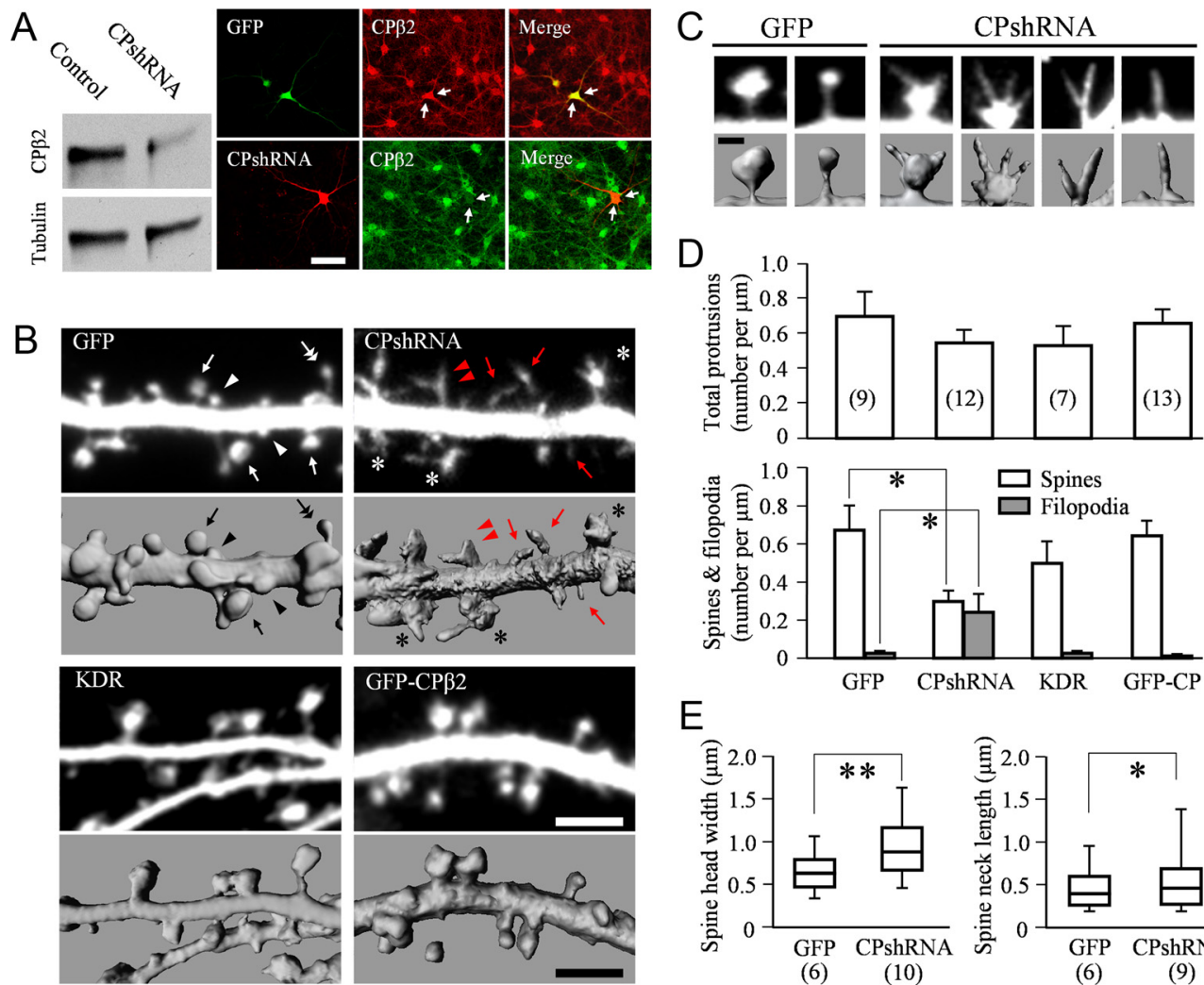

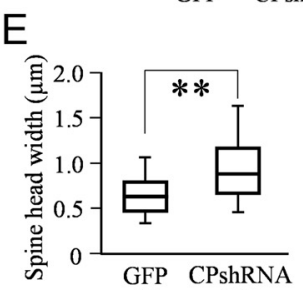

(6) (10)

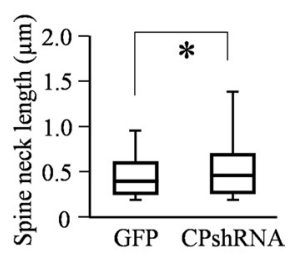

(6) (9)

Figure 2. Knockdown of CP $\beta 2$ by shRNA and its effects on spine development. $A$, Effective knockdown of endogenous CP by CPshRNA in CAD cells (Western blotting) and in hippocampal neurons (immunostaining). Scale bar, $20 \mu \mathrm{m}$. B, Representative images of dendritic protrusions in hippocampal neurons expressing GFP, (PshRNA, KDR, and GFP-CP, each accompanied by its 3 D reconstruction view. Arrows: mushroom spine; arrowheads: stubby; double arrows: thin spines; red arrows: thin filopodia; red arrowheads: branched protrusions; asterisks: spines with protrusions from the head. $S$ Cale bar: $5 \mu \mathrm{m}$. $C$, Representative images of different types of dendritic protrusions in control and (P knockdown neurons (top, fluorescence; bottom, 3D reconstructed view). Scale bar: $1 \mu \mathrm{m}$. $D$, Quantitative analyses of total protrusions and spineand filopodia densities. Error bars represent $95 \%$ confidenceinterval. E, Box-whisker plotshowing the spinehead width and necklength of different groups (bottom and top of the box,25\% and $75 \%$; middle band, $50 \%$; ends, $5 \%$ and $95 \%$ ). Numbers in parentheses indicate the number of neurons analyzed in each group. ${ }^{*} p<0.05$; ${ }^{* *} p<0.01$; ANOVA Dunnett's method for $\boldsymbol{D}$ and Student'st test for $\boldsymbol{E}$.

A

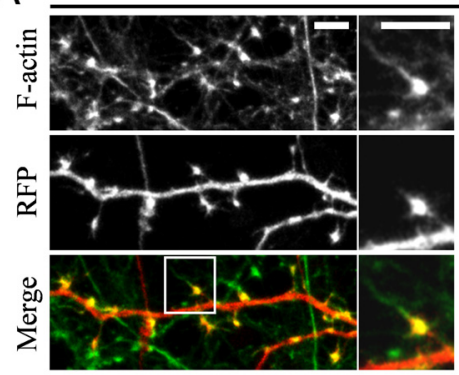

\section{B}

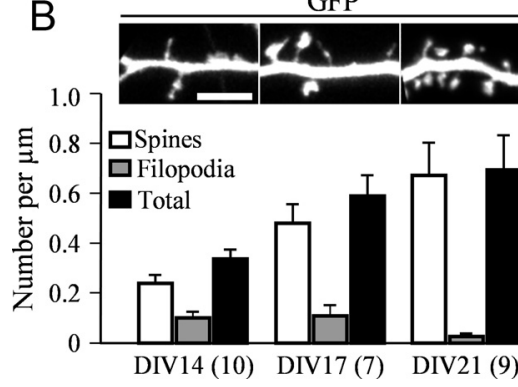

CPshRNA
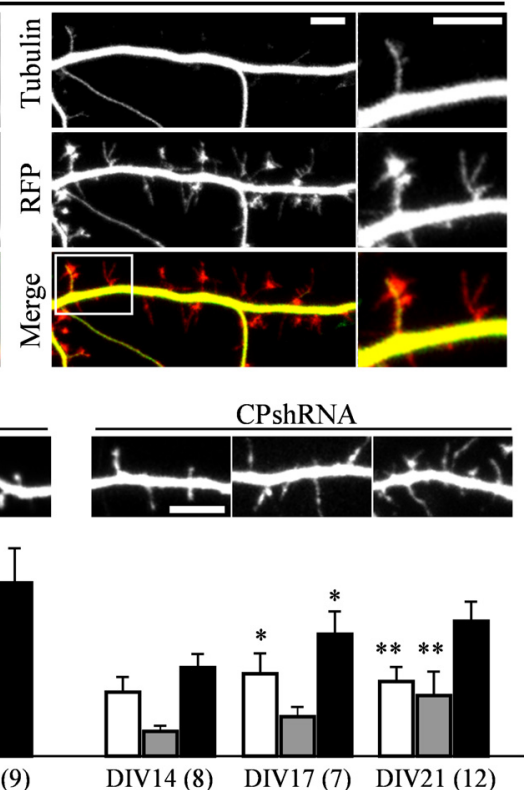

Figure 3. CPknockdown on the cytoskeleton and development of dendritic protrusions. $\boldsymbol{A}$, Representativeimages show theF-actin (labeled by Alexa488-phalloidin; left) and microtubules (labeled by GFP-tubulin; right) in (PshRNA-transfected neurons (indicated by the RFP fluorescence). For eachset, thearea enclosed by the rectangle is shown in a magnified view on the right. $\boldsymbol{B}$, Representativeimages showing the changes in filopodia and spines of cultured hippocampal neuronsatDIV14,DIV17, and DIV21 as revealed by confocal imaging. Bargraph showsthequantitativeanalysis of the number of different dendritic protrusions at different stages in neurons transfected with GFP alone or CPshRNA. The number of neurons analyzed in each group is indicated in parentheses. Errorbars represent $95 \%$ confidence interval. ${ }^{*} p<0.05$; ${ }^{* *} p<0.001$ (Student'sttest). Scale bars, $5 \mu \mathrm{m}$.

and PSD-95 (postsynaptic marker). We found that almost all of the dendritic spines in control cells were apposed by SV2 puncta (Fig. 4A) and contained PSD-95 signals (Fig. $4 B$ ), which is consistent with previous studies showing that most of the spines, if not all, represent the postsynaptic structure of functional synapses. In cells expressing CPshRNA, SV2 signals were also found to be associated with the spine-like protrusions, but the density was markedly reduced (Fig. 4A). Interestingly, the size of the SV2 puncta appears to be larger than that of the control group (Fig. 4A). Similarly, PSD-95 signals were associated with the spine-like protrusions even though these spines have thin filopodia on the spine head (Fig. 4B). The density of PSD-95 puncta was also reduced but their size appeared to larger than that of the control group (Fig. 4B). To better determine the effects of CP knockdown on synaptic function, we performed whole-cell patch-clamp recordings to examine the mEPSCs. We found that the frequency of mEPSCs in cells expressing CPshRNA was markedly reduced compared with the control nontransfected cells (Fig. 4C-E). Expression of the KDR construct restored the 

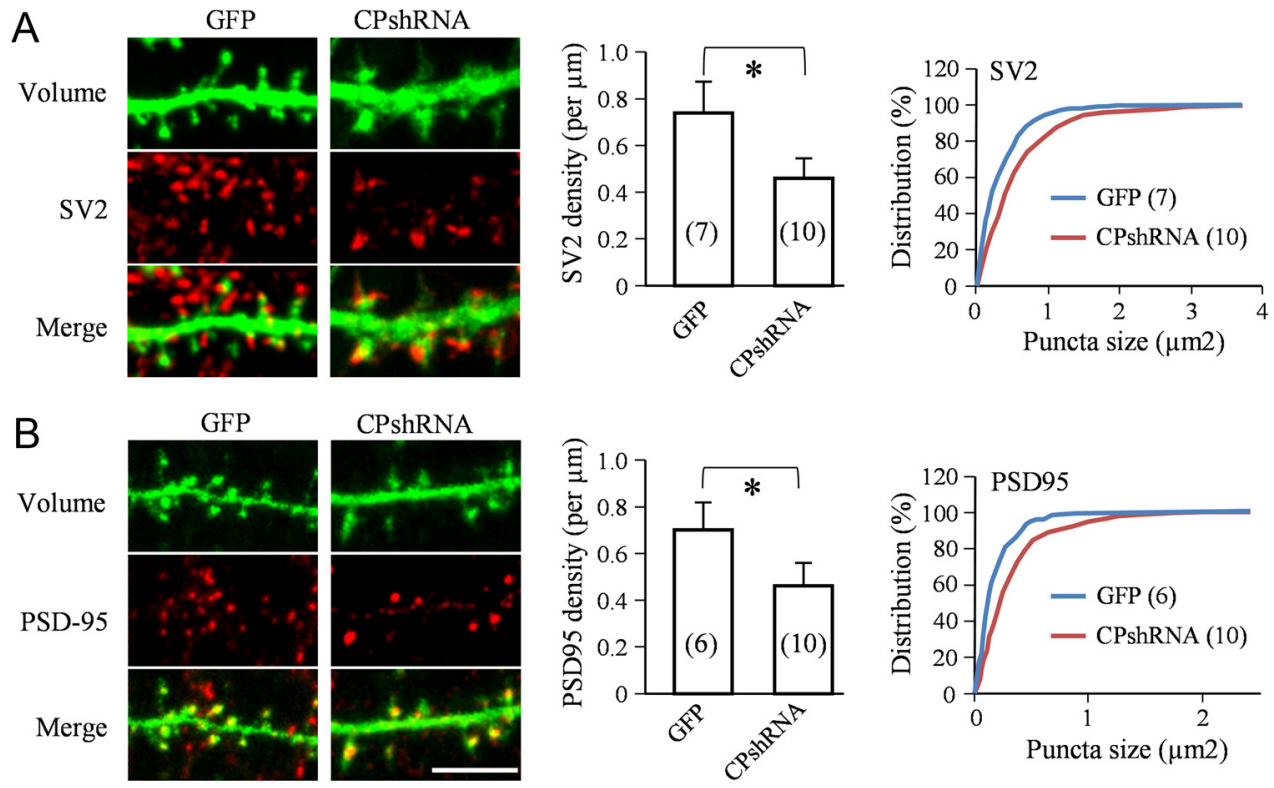
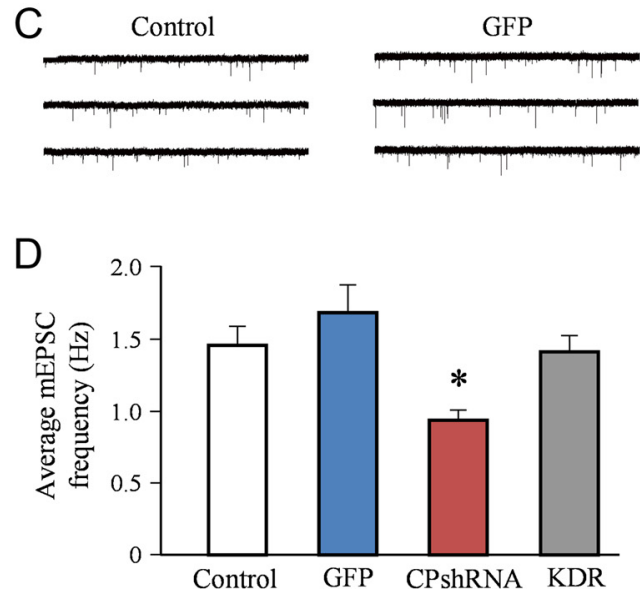
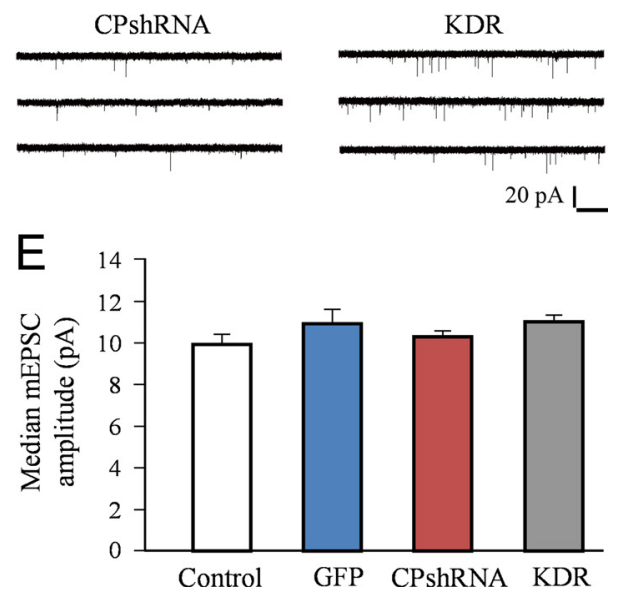

Figure 4. Impaired synapse formation and function by CP knockdown. $\boldsymbol{A}, \boldsymbol{B}$, Effects of CP knockdown on the presynaptic marker SV2 ( $\boldsymbol{A}$ ) and the postsynaptic marker PSD-95 ( $\boldsymbol{B}$ ) as shown by representative images of immunofluorescence (left), the density (center), and the size distribution (right). For (PshRNA-expressing cells, RFP is the volume marker but is displayed in green for visualization purpose. $\boldsymbol{C}-\boldsymbol{E}$, Whole-cell patch-clamp recordings of mEPSCs in nontransfected neurons (control), transfected with GFP alone (GFP), with (PshRNA, and the KDR constructs. Sample traces of mEPSCs are shown in $\boldsymbol{C}$, and quantifications of the frequency and median amplitude are shown in $\boldsymbol{D}$ and $\boldsymbol{E}$, respectively. $n=24,23,44$, and 43 for control, GFP, CPshRNA, and KDR, respectively. ${ }^{*} p<0.01$, Student's $t$ test.

mEPSC frequency, and expression of GFP alone did not have any effect on the mEPSC frequency (Fig. $4 C, D$ ). All of the groups showed similar amplitudes of mEPSCs (Fig. 4E). These electrophysiological data, together with the imaging findings, support a critical role for $\mathrm{CP}$ in spine development and synapse formation.

\section{Discussion}

End capping of cytoskeletal filaments is a key mechanism for regulating filaments' elongation and disassembly, as well as the organization of the cytoskeletal architecture. CP binds to the barbed ends of actin filaments to inhibit further elongation and is involved in the formation of branched actin networks in concert with Arp2/3 (Akin and Mullins, 2008). Here, we show that CP plays an essential role in the formation of dendritic spines. In particular, CP knockdown promoted the formation of thin filopodia-like protrusions and inhibited proper spine development. Dendritic spines initiate as filopodia-like protrusions from dendritic shafts, and then convert to mature spine structure with an expanded head (Ziv and Smith, 1996; Yoshihara et al., 2009; Hotulainen and Hoogenraad, 2010). Our data thus suggest that
$\mathrm{CP}$ may function in the transition of filopodia to spines. $\mathrm{CP}$ is known to function in the formation of branched actin networks in lamellipodia in non-neuronal cells, and CP knockdown promotes the formation of filopodia (Mejillano et al., 2004). Recent studies have revealed that spine heads are supported by a branched actin filamentous network with a core of actin bundles interacting with the PSD. It was postulated that spine head expansion involves extensive actin branching by Arp $2 / 3$ occurring at the tip of filopodia when the spine head begins to form (Hotulainen and Hoogenraad, 2010). Because CP has been shown to function in Arp2/3-mediated nucleation of actin branches (Akin and Mullins, 2008), it is conceivable that CP functions in a similar way to facilitate Arp2/3-mediated actin nucleation and branching for spine head expansion.

The relatively larger and irregularly shaped spine heads and their emergence of thin filopodia in CP knockdown cells also indicate the importance of stopping filament elongation during spine development. It is conceivable that the lack of CP by shRNA knockdown allowed continuous polymerization of actin fila- 
ments in spines, leading to the larger spine heads and filopodial protrusion. The knockdown of CP might have also tilted the balance of the barbed end binding toward Ena/Vasp-mediated actin bundling for filopodial protrusion (Bear et al., 2002). One would thus expect that CP overexpression could favor less filopodia and more spine formation, but EGFP-CP $\beta 2$ expression did not produce any effect on dendritic protrusions. This can be explained by the fact that CP functions as a heterodimer of $\alpha$ and $\beta$ subunits (Cooper and Sept, 2008). The exogenous EGFP-CP $\beta 2$ requires endogenous $\alpha$ subunits to be functional and stable. Therefore, a potential effect on spine formation may only be observed by overexpressing both $\alpha$ and $\beta$ subunits.

The altered spine formation by CP knockdown appears to directly translate into a reduction in the formation of functional synapses. Although the spines with altered shape in CP knockdown neurons were able to form functional synapses, the number of either presynaptic or postsynaptic markers was largely reduced. This is consistent with the reduced spine density in neurons with CP knockdown. It is likely that the thin filopodia-like protrusions may not support postsynaptic specialization required for synapse formation. Indeed, our live cell imaging of pHluorin-tagged GluR1 found that most spines (even with their aberrant shape), but not filopodia, in CP knockdown neurons exhibited a concentrated level of surface GluR1 (data not shown). This conclusion is further supported by the reduced frequency of mEPSCs recorded from the CPshRNA knockdown cells. Although the size of both presynaptic and postsynaptic markers appeared to be slightly larger in CP knockdown neurons, no difference was observed in the amplitude of mEPSCs between the control and CP knockdown groups, suggesting that the number of receptors at the postsynaptic site was not substantially altered by CP knockdown. Together with the imaging data, CP appears to play a prominent role in converting filopodia to spines for functional synapses. Whether CP is also involved in spine dynamics and receptor trafficking during synaptic plasticity remains to be investigated.

Our findings on the role of CP in synapse formation and function are supported by several studies implicating CP in brain functions and disorders. For example, CP interaction with tubulin was found to be altered in hippocampi of rats exposed to a spatial memory task (Nelson et al., 2004), although our data do not show altered microtubule presence in spines after CP knockdown. In fetal brains of Down syndrome, a frequently inherited disease causing mental retardation, both $\mathrm{CP} \alpha$ and $\mathrm{CP} \beta$ levels were significantly lower than the control (Gulesserian et al., 2002). Moreover, CP level was found to decrease in a rat model of dementia, and CP accumulation in synapses was observed after LTP stimulation (Kitanishi et al., 2010). Together, it is clear that proper actin organization and remodeling in the dendritic spines are crucial for the development and maturation of the postsynaptic structure and function. Alterations of the spine actin structure and its dynamic regulation likely present a mechanism underlying abnormality in synapse development and function found in many brain disorders.

\section{References}

Akin O, Mullins RD (2008) Capping protein increases the rate of actinbased motility by promoting filament nucleation by the Arp $2 / 3$ complex. Cell 133:841-851.

Bear JE, Svitkina TM, Krause M, Schafer DA, Loureiro JJ, Strasser GA, Maly IV, Chaga OY, Cooper JA, Borisy GG, Gertler FB (2002) Antagonism between Ena/VASP proteins and actin filament capping regulates fibroblast motility. Cell 109:509-521.
Carlisle HJ, Kennedy MB (2005) Spine architecture and synaptic plasticity. Trends Neurosci 28:182-187.

Cingolani LA, Goda Y (2008) Actin in action: the interplay between the actin cytoskeleton and synaptic efficacy. Nat Rev Neurosci 9:344-356.

Cooper JA, Sept D (2008) New insights into mechanism and regulation of actin capping protein. Int Rev Cell Mol Biol 267:183-206.

Deng L, Yao J, Fang C, Dong N, Luscher B, Chen G (2007) Sequential postsynaptic maturation governs the temporal order of GABAergic and glutamatergic synaptogenesis in rat embryonic cultures. J Neurosci $27: 10860-10869$.

Fiala JC, Feinberg M, Popov V, Harris KM (1998) Synaptogenesis via dendritic filopodia in developing hippocampal area CA1. J Neurosci 18:8900-8911.

Gu J, Firestein BL, Zheng JQ (2008) Microtubules in dendritic spine development. J Neurosci 28:12120-12124.

Gu J, Lee CW, Fan Y, Komlos D, Tang X, Sun C, Yu K, Hartzell HC, Chen G, Bamburg JR, Zheng JQ (2010) ADF/cofilin-mediated actin dynamics regulate AMPA receptor trafficking during synaptic plasticity. Nat Neurosci 13:1208-1215.

Gulesserian T, Kim SH, Fountoulakis M, Lubec G (2002) Aberrant expression of centractin and capping proteins, integral constituents of the dynactin complex, in fetal down syndrome brain. Biochem Biophys Res Commun 291:62-67.

Hart MC, Korshunova YO, Cooper JA (1997) Vertebrates have conserved capping protein alpha isoforms with specific expression patterns. Cell Motil Cytoskeleton 38:120-132.

Hotulainen P, Hoogenraad CC (2010) Actin in dendritic spines: connecting dynamics to function. J Cell Biol 189:619-629.

Hotulainen P, Llano O, Smirnov S, Tanhuanpää K, Faix J, Rivera C, Lappalainen P (2009) Defining mechanisms of actin polymerization and depolymerization during dendritic spine morphogenesis. J Cell Biol 185:323-339.

Hu X, Viesselmann C, Nam S, Merriam E, Dent EW (2008) Activitydependent dynamic microtubule invasion of dendritic spines. J Neurosci 28:13094-13105.

Kasai H, Matsuzaki M, Noguchi J, Yasumatsu N, Nakahara H (2003) Structure-stability-function relationships of dendritic spines. Trends Neurosci 26:360-368.

Kitanishi T, Sakai J, Kojima S, Saitoh Y, Inokuchi K, Fukaya M, Watanabe M, Matsuki N, Yamada MK (2010) Activity-dependent localization in spines of the F-actin capping protein CapZ screened in a rat model of dementia. Genes Cells 15:737-747.

Korobova F, Svitkina T (2010) Molecular architecture of synaptic actin cytoskeleton in hippocampal neurons reveals a mechanism of dendritic spine morphogenesis. Mol Biol Cell 21:165-176.

Mejillano MR, Kojima S, Applewhite DA, Gertler FB, Svitkina TM, Borisy GG (2004) Lamellipodial versus filopodial mode of the actin nanomachinery: pivotal role of the filament barbed end. Cell 118:363-373.

Nelson TJ, Backlund PS Jr, Alkon DL (2004) Hippocampal protein-protein interactions in spatial memory. Hippocampus 14:46-57.

Qi Y, Wang JK, McMillian M, Chikaraishi DM (1997) Characterization of a CNS cell line, CAD, in which morphological differentiation is initiated by serum deprivation. J Neurosci 17:1217-1225.

Schafer DA, Korshunova YO, Schroer TA, Cooper JA (1994) Differential localization and sequence analysis of capping protein beta-subunit isoforms of vertebrates. J Cell Biol 127:453-465.

Tada T, Sheng M (2006) Molecular mechanisms of dendritic spine morphogenesis. Curr Opin Neurobiol 16:95-101.

van Spronsen M, Hoogenraad CC (2010) Synapse pathology in psychiatric and neurologic disease. Curr Neurol Neurosci Rep 10:207-214.

Vitriol EA, Uetrecht AC, Shen F, Jacobson K, Bear JE (2007) Enhanced EGFP-chromophore-assisted laser inactivation using deficient cells rescued with functional EGFP-fusion proteins. Proc Natl Acad Sci U S A 104:6702-6707.

Yoshihara Y, De Roo M, Muller D (2009) Dendritic spine formation and stabilization. Curr Opin Neurobiol 19:146-153.

Yuste R, Bonhoeffer T (2001) Morphological changes in dendritic spines associated with long-term synaptic plasticity. Annu Rev Neurosci 24:1071-1089.

Ziv NE, Smith SJ (1996) Evidence for a role of dendritic filopodia in synaptogenesis and spine formation. Neuron 17:91-102. 